\title{
Trends und Herausforderungen im Gesundheitswesen der Schweiz
}

\section{Peter C. Meyer ${ }^{a}$, Mathis Brauchbarb}

a Prof. Dr. phil., selbständiger Berater, Zürich; bis 2016 Direktor Departement Gesundheit, ZHAW Zürcher Hochschule für Angewandte Wissenschaften, Winterthur; 'b Partner der Advocacy AG, Zürich

Das Gesundheitswesen einzelner Länder ist zahlreichen nationalen und internationalen Einflüssen ausgesetzt. Sie bestimmen mit, wie sich das Gesundheitssystem und das Gesundheitswesen in den kommenden Jahren entwickeln wird. Der Artikel beschreibt die wichtigsten medizinischen, technischen und sozialen Trends.

\section{Demographische Alterung und Zunahme chronischer Krankheiten}

Seit Mitte des 19. Jahrhunderts nimmt der Anteil der chronischen Krankheiten in der Gesundheitsversorgung und als Todesursache laufend zu. Gleichzeitig wird die Bevölkerung immer älter, und dieser Trend wird auch in den kommenden Jahrzehnten weiter anhalten. Neben dem Alter sind Lebensbedingungen und Lebensstile wichtige Determinanten chronischer Erkrankungen. Mit der Alterung der Gesellschaft und der damit verbundenen Zunahme chronischer Erkrankungen wächst auch der Anteil jener Menschen in der Schweiz, die auf Pflege und Therapie angewiesen sind.

\section{Résumé}

Le secteur de la santé des différents pays est soumis à de nombreuses influences nationales et internationales. Celles-ci co-déterminent l'évolution du système et du secteur de la santé au cours des années suivantes. L'article décrit les principales tendances médicales, techniques et sociales. Les contradictions et les conflits associés aux tendances s'expriment souvent sous la forme de contre-tendances. De ces tendances et contretendances, il est possible de déduire l'évolution future du système de santé suisse. II en ressort que celui-ci continuera à s'étendre au cours des prochaines décennies. Mais il sera plus intégré, préventif, prédictif, ambulatoire, transparent et axé sur les données. Les métiers de la santé, en particulier, doivent désormais anticiper cette évolution et développer leurs profils professionnels et leurs compétences en conséquence.

\section{Entwicklungen und Trends}

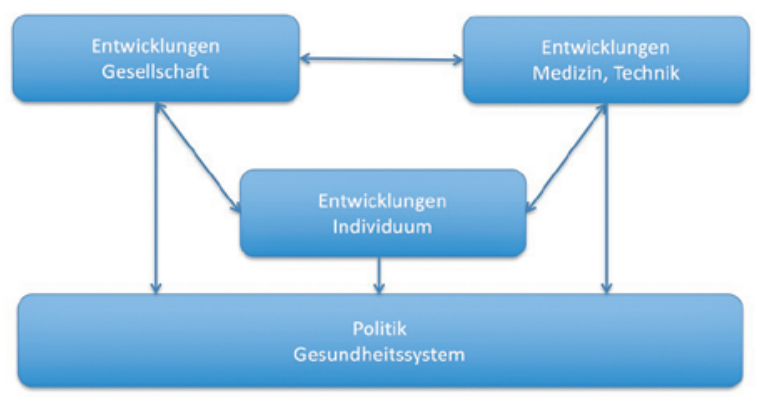

Abbildung 1: Systemische Betrachtung von Entwicklungen.

Trend 1: Personalisierte Medizin

Die rasch um sich greifende Digitalisierung unseres Lebens und die Fortschritte in den biologischen Wissenschaften, insbesondere in der Genomik und der Proteomik, führen in den nächsten zehn Jahren zu einer Personalisierung der Medizin. So kann die Gesundheitsversorgung spezifischer auf das Individuum abgestimmt werden. Prädiktiv wird die personalisierte Medizin, wenn die individuellen Gesundheitsdaten bereits in einem gesunden Stadium erhoben und mit Bevölkerungsdaten verknüpft werden. Dies erlaubt es, individuelle Risiken zu errechnen und entsprechende präventive Massnahmen frühzeitig zu ergreifen.

\section{Gegentrend 1: Traditionelle Medizin}

Auf der Ebene der Bevölkerung werden sich als Reaktion wertkonservative und technologiekritische Personen der personalisierten Medizin gegenüber abstinent verhalten. Insbesondere die prädiktiven Aspekte werden von ihnen abgelehnt. 
Trend 2: Digitale Medizin

Gemäss Eric Topol wird die Digitalisierung die Gesundheitsversorgung fundamental verändern [1]. Topol geht dabei von einer sogenannten Superkonvergenz aus: Internet, mobile Kommunikation, Rechenleistungen und Social Networking erlauben es, die Daten aus medizinischer Praxis, Bildgebung, Bioanalytik, aus Gesundheitsinformationssystemen und mobilem Monitoring (Sensoren, Applikationen) zu verknüpfen, zu aggregieren und zu analysieren. Im Sinne eines digitalen Mutterpasses können alle Daten gespeichert und bei Bedarf von allen bei der Betreuung beteiligten Berufsgruppen gelesen und ergänzt werden. Der Anpassungsbedarf im Gesundheitssystem ist in der Folge enorm. Insbesondere die Arzt-Patienten-Beziehung wird dadurch stark verändert [2]. Der Arzt wird vermehrt als Coach und Berater tätig sein, das ärztliche Diagnosemonopol dürfte fallen.

Das Individuum ist stärker als bisher an der Datenerhebung, Forschung, Diagnose und Entscheidungsfindung sowie auch an der Behandlung beteiligt. Dies wird die Position der Patientinnen und Patienten gegenüber Spitälern, Ärztinnen, Ärzten und auch Versicherungen erheblich stärken.

\section{Gegentrend 2: Erfahrungs- und Komplementär- medizin}

Neben einer digitalen und evidenzbasierten Medizin wird die Erfahrungs- und Komplementärmedizin im Bereich der chronischen Erkrankungen und der Gesundheitsförderung weiterhin grossen Zulauf haben. Die Patienten werden dabei weiterhin das aus ihrer Sicht Beste aus diesen beiden gegensätzlichen «Welten» kombinieren.

\section{Trend 3: Robotik und Automatisierung}

Repetitive, schwere und hochpräzise Arbeiten werden zunehmend durch Roboter und automatisierte Systeme verrichtet. Im Operationssaal sind Roboter als Präzisionswerkzeuge längst Alltag. In der Rehabilitation werden zunehmend intelligente Mensch-Maschinen-Systeme verwendet. Auch in der Pflege werden Roboter eingesetzt: Bei Routinehandlungen und schweren Verrichtungen werden sie das Pflegepersonal stark entlasten. Sie werden aber auch Betreuungsaufgaben übernehmen, etwa als Begleiter von Alzheimer-Patienten.

\section{Gegentrend 3: Mensch statt Maschine}

Vor allem der Einsatz von Robotern in Pflege, Rehabilitation und Betreuung ruft Widerspruch hervor. Die Automaten werden einerseits als Jobkiller, andererseits als Beleg einer zunehmend unmenschlichen
Behandlung Kranker und Bedürftiger moralisch betrachtet. In der Folge wird das Menschliche in Pflege, Rehabilitation und Betreuung vermehrt betont. Roboterfreie Angebote werden aber fast nur finanziell besser gestellten Menschen zugänglich bleiben.

\section{Trend 4: Medikalisierung}

In den vergangenen Jahrhunderten war Gesundheit ein Sekundärwert: Gesundheit hatte zum Zweck, einen höheren persönlichen oder gesellschaftlichen Wert zu erreichen (Arbeit, gottgefälliges Leben etc.). Seit Ende des 20. Jahrhunderts ist die Gesundheit zu einem Wert per se geworden. Gesundheit als Wert dominiert weite Lebensbereiche. Dies befördert auch eine Medikalisierung: Der Krankheitsbegriff wird weiter ausgeweitet [3] In der Folge werden bisher als normal geltende Verhalten und Empfindungen sowie Normabweichungen, die als gesellschaftlich problematisch gelten, medizinisch gedeutet und entsprechend behandelt (z.B. Trauer, Bewegungsdrang bei Kindern und Erwachsenen [ADHS], chronischer Reizdarm) [4]. Die Medikalisierung führt letztlich zu einer Zunahme von Prävention, Diagnosen und Behandlungen und damit zu einer Ausweitung des Einflussbereiches der Medizin.

\section{Gegentrend 4: Hedonismus, Diversity und "natural health»}

Ein bemerkenswerter Gegentrend ist die lustbetonte, hedonistische Einstellung eines Bevölkerungsteils, welche dem kontrollierten und medikalisierten Leben der Mehrheit ein bewusst ungesundes beziehungsweise unkontrolliertes Gesundheitsverhalten entgegensetzt. Weitere Gegentrends sind das Leitbild Diversity, das zu einer erhöhten Toleranz gegenüber abweichendem Verhalten führt, und das natural-health movement, das eine «natürliche» Gesundheit bei möglichst wenig professionellen Interventionen fördern will.

\section{Trend 5: Selbstmanagement und Selbst-Monitoring} Zahlreiche Studien belegen, dass sich eine aktive Mitwirkung des Patienten an der Therapie bei chronischen Erkrankungen positiv auf den Verlauf auswirkt [5]. Bei der Behandlung mehrerer Krankheiten ist das Selbstmanagement bereits fortgeschritten, z.B. bei Diabetes, bei Morbus Bechterew und bei chronisch obstruktiver Lungenerkrankung (COPD). Aufgrund neuerer Technologien bei der Überwachung und der Anwendung von Therapien werden aber auch andere chronische Erkrankungen inklusive der psychischen Erkrankungen künftig viel stärker durch die Patienten selbst gesteuert. Dies ermöglicht chronisch kranken und betagten Menschen, länger selbständig zu Hause zu leben. 


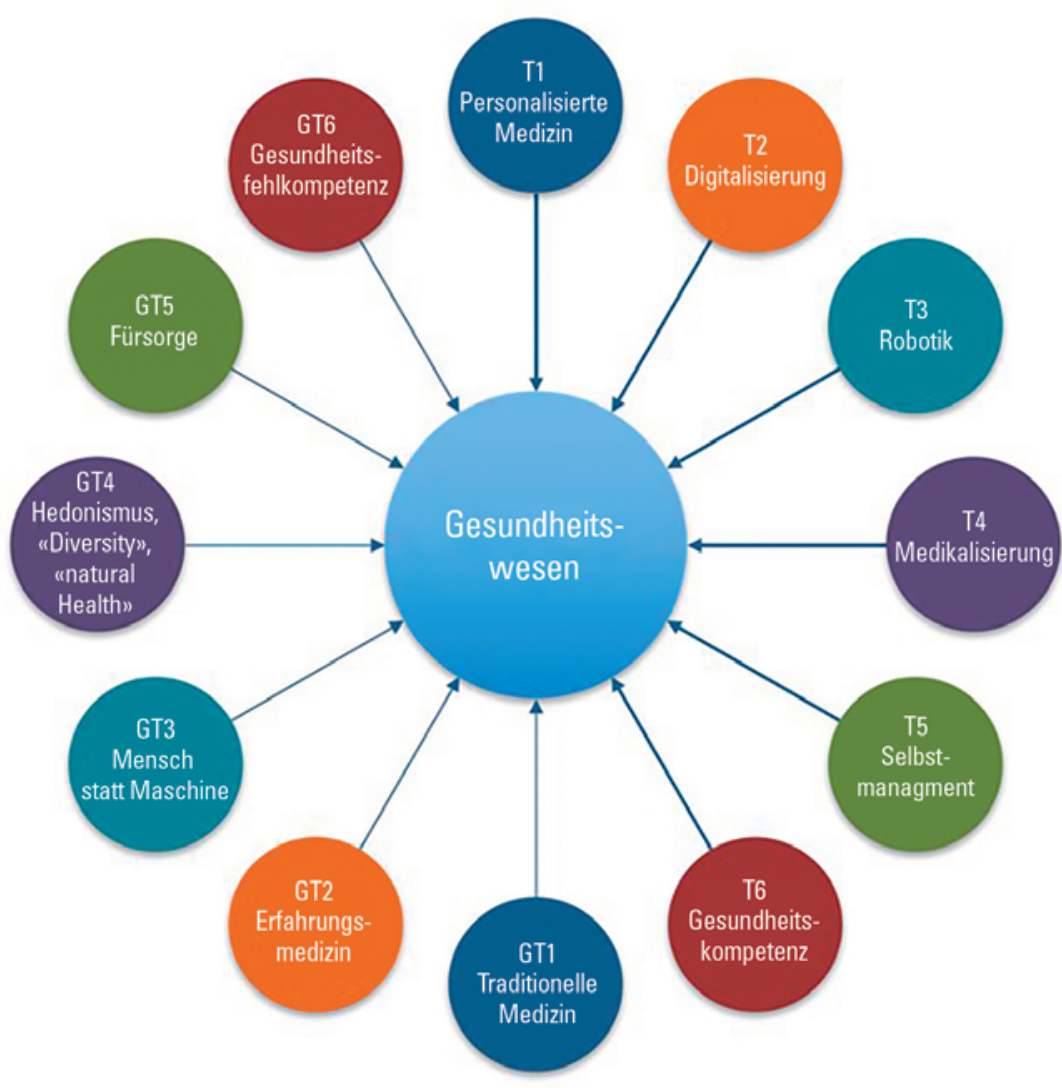

Abbildung 2: Trends und Gegentrends. pliance) sowie von der Einfachheit und der sozialen Akzeptanz der technischen Hilfsmittel [7]. Da sich Gesundheitsinformationen mehr und mehr um Zahlen und Risiken drehen (chronische Krankheiten), gehört zur Gesundheitskompetenz nicht nur ein Sach-, sondern auch ein Zahlenverständnis (Numeracy), also die Fähigkeit, beispielsweise Resultate aus Laboruntersuchungen und Evaluationsstudien korrekt interpretieren zu können [8].

\section{Gegentrend 6: Gesundheitsfehlkompetenz}

Die vermehrten Informationsmöglichkeiten (Internet, Social Media) können zwar zu besseren Gesundheitskompetenzen beitragen und damit zu mehr Selbstbestimmung der Patienten führen, enthalten allerdings viele falsche und einseitige Informationen, was wiederum eine falsche Aufklärung und Halbwissen fördert.

\section{Auswirkungen auf das Gesundheits- system}

\section{Effizienzsteigerung}

Der massive Ausgabenanstieg im gesamten Gesundheitswesen ist etwa zu gleichen Teilen demographischen und nicht demographischen Kostentreibern geschuldet (gestiegene Ansprüche der Bevölkerung, Medikalisierung und medizinisch-technischer Fortschritt). Die Belastung der öffentlichen und der privaten Haushalte wird dementsprechend zunehmen, ebenso der Kostendruck, also die Tendenz, möglichst effizient mit den finanziellen Ressourcen umzugehen In diesem Umfeld werden technische Trends wie die Digitalisierung und die Robotik gefördert, da sie eine Effizienzsteigerung versprechen.

Angesichts des zunehmenden Kostendrucks wird die Orientierung an der medizinischen Evidenz weiter zunehmen. Kostenträger sind nur noch bereit, Behandlungen zu vergüten, die auf Evidenz basieren und auf davon abgeleiteten Richtlinien. Im Zuge der Entwicklung neuer Qualitätsinstrumente und der Kostendiskussion werden Managed Care-Modelle sich weiter ausbreiten.

\section{Integrierte Versorgung}

Es werden vermehrt integrierte Angebote entstehen, die in letzter Konsequenz die gesamte Kette der Interventionen abdecken: Prävention - ambulante Versorgung - stationäre Akutversorgung - Rehabilitation Langzeitversorgung.

Primär-, Sekundär- und Tertiärprävention werden weiterhin an Bedeutung gewinnen, u.a. weil integrierte Angebote und Managed Care-Modelle zunehmen wer- 
den. Wahrscheinlich ist, dass Gesundheitsförderung und Prävention gerade in Hinblick auf chronische Erkrankungen nicht mehr getrennt betrachtet und angeboten werden, sondern dass sich auch hier ein integrierter Ansatz durchsetzt [9].

\section{Neue Angebote}

Die Ambulantisierung verlagert Versorgungsleistungen aus dem stationären in den ambulanten Sektor. Die 2012 erfolgte obligatorische Einführung der Fallpauschalen-Vergütung (SwissDRG) in den Schweizer Spitälern verstärkt die Verkürzung der stationären Behandlung. Das Zuhause ist in vielen Fällen eine kostengünstige Alternative zum Spital- und Heimaufenthalt.

Das Zuhause wird auch in der Langzeitpflege in den nächsten Jahrzehnten weiter an Bedeutung gewinnen [10]. Dazu tragen einerseits der Kostendruck und die demographische Entwicklung bei. Andererseits werden die Angebote in der Fernüberwachung und in der mobilen Kommunikation weiterentwickelt. Man spricht auch von einem anstehenden Boom von Überwachungs-, Diagnose- und Behandlungsangeboten zu Hause. Der Markt für den Verkauf und den Verleih sogenannter Home Medical Equipments wird stark wachsen, und damit entsteht auch zusätzlicher Bedarf an Beratung.

\section{Qualität}

Um die Qualität in der Versorgung sicherzustellen und laufend zu verbessern, werden die Behandlungsdaten der einzelnen Leistungserbringer in der Form von Outcome-Daten kontinuierlich erhoben und einem Benchmarking unterzogen. Von Seiten der Patienten und der Versicherten wird der Druck zunehmen, die OutcomeDaten der einzelnen Leistungserbringer zu veröffentlichen und miteinander zu vergleichen [11]. Dadurch wird es erst möglich sein, die Wahlfreiheit in der Behandlung konsequent zu nutzen.

\section{Neue Berufsbilder}

Die Gesundheitsberufe stehen heute unter einem hohen Erneuerungsdruck. In den nächsten Jahren und Jahrzehnten werden neue Berufsbilder und neue Berufsprofile entwickelt. Historisch gewachsene und etablierte Grenzen zwischen Tätigkeitsgebieten, die zurzeit durch die Gesundheitsberufe besetzt werden, werden zerfliessen.

So wird die bestehende Lücke zwischen aufwendig universitär ausgebildeten Ärzten und den weiteren Gesundheitsberufen durch weitere Berufsbilder ge- schlossen. Dies können einerseits Ärzte mit verkürztem Studium sein oder Pflege- und Therapieberufe mit ergänztem Studium und Zusatzkompetenzen (Advanced Practice, Physician Assistant). Die Gesundheitsberufe werden nicht nur im kurativen Bereich tätig sein, sondern vermehrt auch im Bereich der Prävention und Gesundheitsförderung. Dies bedeutet auch neue Einsatzorte und Kontexte wie z.B. Wirtschaftsbetriebe, Kindergärten und Schulen.

\section{Fazit}

Aufgrund der beschriebenen Entwicklungen wird das Gesundheitssystem weiter wachsen und sich laufend verändern. Das Gesundheitssystem wird vermehrt integriert, präventiv, prädiktiv, ambulant, transparent und datengetrieben sein. Da ein Teil der Bevölkerung und der Health Professionals die dominanten Trends ablehnen, werden neue Formen der Ungleichheit entstehen, und die Variationen des Gesundheits- und Krankheitsverhaltens werden zunehmen. Die Gesundheitsberufe müssen diese Entwicklung antizipieren und ihre Berufsbilder und Kompetenzen entsprechend weiterentwickeln.

Interessenverbindungen

Es bestehen keine Interessenverbindungen der Autoren, die diesen Artikel betreffen könnten.

Bildnachweis

Abbildungen von Autoren erstellt

Literatur

1 Topol E. The Creative Destruction of Medicine: How the Digital Revolution Will Create Better Health Care. New York: Basic Books; 2012

2 Topol E. The Patient will see you now: The Future of Medicine is in your Hands. New York: Basic Books; 2015

3 Meyer PC. Gesellschaftliche Hintergründe der Medikalisierung. Bioethica Forum. 2012;5(2):52-8.

4 Conrad P. The Medicalization of Society. Baltimore: John Hopkins University Press; 2007

5 Brownson CA, et al. Cost-effectiveness of diabetes self-management programs in community primary care settings. Diabetes Educ. 2009;35(5):761-9

6 In der Schweiz u.a.: https://www.dacadoo.com

7 Nutbeam D. Health Literacy as a Public Health Goal. Health Prom Int. 2000;15(3):259-67.

8 Rothman RL. The Role of Numeracy in Health Care. J Health Commun. 2008;13(6):583-95.

9 Steiger D, Brauchbar M, Brauchbar D. Prävention in der Gesundheitsversorgung verankern: Zentrale Dimensionen und Case Studies. Bern: Studie im Auftrag des Bundesamtes für Gesundheit 2016

10 Shepperd S, Wee B, Straus SE. Hospital at home: home-based end of life care. Cochrane Database Syst Rev. 2011;7. doi:10.1002/14651858. CD009231.

11 Olmsted Teisberg E. Nutzenorientierter Wettbewerb im Schweizer Gesundheitswesen. Zürich/Basel/Bern: economiesuisse, Klinik Hirslanden AG, Interpharma, Schweizerischer Versicherungsverband SVV, Swisscom IT Services AG; 2008. 